\title{
Position Optimization with Spectrum Sensing Decision for Unmanned Aerial Vehicles
}

\section{Zinan Guo ( $\nabla$ davide_guo@bupt.edu.cn )}

Beijing University of Posts and Telecommunications

\section{Liuying Wang}

Xidian University

\section{Research}

Keywords: Unmanned Aerial Vehicle, spectrum sensing, unless outage probability

Posted Date: September 13th, 2021

DOI: https://doi.org/10.21203/rs.3.rs-889632/v1

License: (c) (i) This work is licensed under a Creative Commons Attribution 4.0 International License. Read Full License 


\title{
Position Optimization with Spectrum Sensing Decision for Unmanned Aerial Vehicles
}

\author{
Zinan Guo ${ }^{1 *}$ and Liuying Wang ${ }^{2}$
}

\begin{abstract}
In the emergency scenario of Unmanned Aerial Vehicle (UAV) relay, link interruption caused by shortage of communication resources often occurs. Using spectrum sensing, UAV can expand communication bandwidth and ensure communication quality. However, spectrum sensing of UAV will increase energy consumption and reduce UAV dwell time. Aiming at the contradiction of energy consumption and communication quality, this paper proposes a relay sensing decision algorithm. Firstly, the spectrum sensing model and algorithm are established. Then, the outage probability is taken as the threshold value adjusting three-dimensional coordinates in the UAV relay sensing decision algorithm. Finally, we construct a UAV relay sensing decision algorithm, which adds the limit of unless outage probability threshold to the firefly algorithm to adjust the position of UAV. By adjusting the three-dimensional coordinates of UAV, the unless outage probability is guaranteed for UAV relay link. Through the simulation of building emergency fire scene, it is verified that the UAV relay sensing decision algorithm can automatically find the optimal UAV sensing position and ensure communication quality.
\end{abstract}

Keywords: Unmanned Aerial Vehicle; spectrum sensing; unless outage probability

\section{Introduction}

UAV has the characteristics of flexible deployment, low cost, strong adaptability, and so on, which makes it widely

"Correspondence: davide_guo@bupt.edu.cn

${ }^{1}$ Beijing University of Posts and Telecommunications Beijing, the State Key Laboratory of Networking and Switching Technology, Beijing, China

Full list of author information is available at the end of the article used in the harsh workplace, so as to effectively avoid casualties [1]. UAV is an ideal choice for relay communication due to its high flexibility [2]. In the scene of emergency fire rescue, UAV as a relay is an essential intermediate link of rescue communication for the contact between rescue personnel and emergency headquarters [3]. Although UAV has many advantages in wireless relay communication, the relay communication is easily outage by external interference. The interference may come from a bad environment or channel interference. In order to ensure that no matter what kind of environment the UAV is in, the relay sensing decision algorithm of the UAV is proposed to ensure that the connection is not outage between rescue personnel and emergency command.

UAV sensing is based on cognitive radio (CR) and cognitive radio network (CRN) [4] [5]. In CR and CRN, users are divided into primary user (PU) and secondary user (SU) due to the dynamic spectrum position principle. PU is also called authorized user or primary user, SU is also called cognitive user or unauthorized user. In cognitive radio, spectrum sensing technology is a key issue in the development of $\mathrm{CR}$ and $\mathrm{CRN}$, because users with spectrum sensing functions can use spectrum sensing technology to detect communication channels [6]. In the communication channel, in addition to the interference of noise and other users, it is the first step to use spectrum sensing to judge whether PU has this problem. The combination of cognitive radio and UAV can effectively improve the spectrum efficiency of UAV [7]. The UAV equipped with a CR sensor continuously perceives the surrounding wireless environment and opportunistically accesses the authorized spectrum of the primary user, which solves the problem that the rescue personnel cannot communicate with the emergency headquarters in the emergency scene. After sensing, the UAV evaluates the outage probability of sensing access link, modifies the position of the UAV, and ensures the stability of the relay link.

Paper [8] presents a grid-based design of a primary exclusive region (PER) for spectrum sharing in the 3D unmanned aerial vehicle (UAV) networks to avoid harmful interference with the primary system. Although cognitive radio solves the problem of interference, the selection of forwarding protocol is very important for UAV as the relay node. Paper [9] propose a cooperative secrecy transmission mechanism based on decode and forward protocol to take 
advantage of the transmitter signal of PU as a dedicated radio frequency $(\mathrm{RF})$. As a relay, UAVs usually use outage probability to evaluate the link security. The paper [10] considers that the system asymptotic secrecy outage probability demonstrates the secrecy diversity order and secrecy diversity gains of the UAV assisted relay cognitive network under the Nakagami-m channel. The most important issue in cognitive radio networks is to accurately detect the empty spectrum. Paper [11] determine the empty spectrums by using spectrum sensing methods. In addition to detecting the empty spectrums, spectrum sensing methods can also detect whether one frequency in the spectrum is occupied by the PU. Nowadays, there are many types of research on UAV sensing and relay forwarding, but there are few types of research on adjusting UAV position according to sensing decision to ensure outage probability. In this paper, according to the UAV relay sense to find the communication frequency of rescue personnel, and then evaluate the outage probability at this time, finally use the firefly algorithm to adjust the UAV position to reduce the outage probability.

The main contributions of this paper are as follows

- The spectrum sensing model of the UAV relay and the detecting user's existence algorithm based on the cyclic spectrum are established.

- The outage probability model of the UAV relay link is established. Then combined with the firefly algorithm, the UAV relay sensing decision algorithm is given.

- The UAV relay sensing decision algorithm is used to adjust the UAV position and ensure that the UAV relay communication link is not outage.

The rest of this paper is organized as follows. The definition and introduction of UAV spectrum sensing is presented in section I. Emergency scenario is analyzed and UAV relay sensing decision algorithm is discussed in section II. Results and discussion is shown in section III. Concluding remarks are presented in section IV.

\section{Methods}

In the scene of disaster relief, UAV is used as a communication relay to ensure the communication between rescue personnel and headquarters. For example, in the fire scene, the contents of communication transmission include 1. Video resources generated by video tools carried by rescue workers. 2 . The emergency headquarters shall give orders to the rescue personnel according to the site conditions. In order to maintain the stability of communication, before the rescuers enter the accident site, it is necessary to check the available communication spectrum in the area as an alternative frequency set. When the rescue personnel is in the accident area, the current communication frequency may be disturbed due to the bad environment. At this time, the rescue personnel can not upload the scene video information and receive the command of the headquarters. At the same time, due to the dispersion of rescue personnel, members can not communicate with each other about the frequency to be switched, and can not unify the communication frequency. In this case of signal loss, UAV uses cyclostationary feature detection technology to detect the available standby frequency in the current area and judge which standby frequency the rescuers switch to, so as to establish the communication between the accident site and the emergency headquarters.

In order to solve this problem, it is necessary to add cyclostationarity detection algorithm to UAV and install sensing devices on UAV to realize multi-user and multifrequency communication. Firstly, a spectrum sensing algorithm is used to analyze the spectrum in the region. By analyzing the alternative frequency characteristics in the region, which frequency the user is on, the noise does not have cyclostationarity. The specific algorithm analysis is in the third chapter. In the disaster relief scene, before the rescuers enter the disaster area, the available spectrum in the area is calculated by the ground spectrum sensing equipment, and the optional frequency sequence of the spectrum is established. Then, the rescuers choose the same frequency before entering the disaster area. When the communication is not smooth in the disaster area, they can switch the communication frequency freely. Then, when the headquarters cannot contact the rescue personnel, the UAV is informed to detect the cyclostationary characteristics of the predetermined spectrum and find the communication frequency of the rescue personnel. Because the rescue personnel may be dispersed for some reason, the UAV needs to conduct one-to-one communication connections to all possible frequencies, as shown in figure 1. Finally, when the UAV establishes a connection with the rescue personnel, the UAV informs the headquarters of the communication frequency to establish the connection between the headquarters and the rescue personnel.

The main contents of the paper are as follows. In the process of communication between UAV and users, it will inevitably be affected by the external intentional or unintentional electromagnetic interference. When the interference is too strong, the current communication frequency can not be used. How to choose the available communication frequency is the first problem to be solved in this paper. Cyclostationary detection is a method to judge whether there is a primary user in the current communication frequency. When the detected frequency has users, the UAV will establish a communication channel with the headquarters, otherwise, the next frequency will be detected. Adding cyclostationary detection algorithm to UAV will increase the energy consumption of UAV. How to reduce the energy consumption of UAV is the second problem. Using spectrum sensing technology can get an available spectrum selection sequence, there is a problem of detection threshold setting and detection success probability, a reasonable threshold 


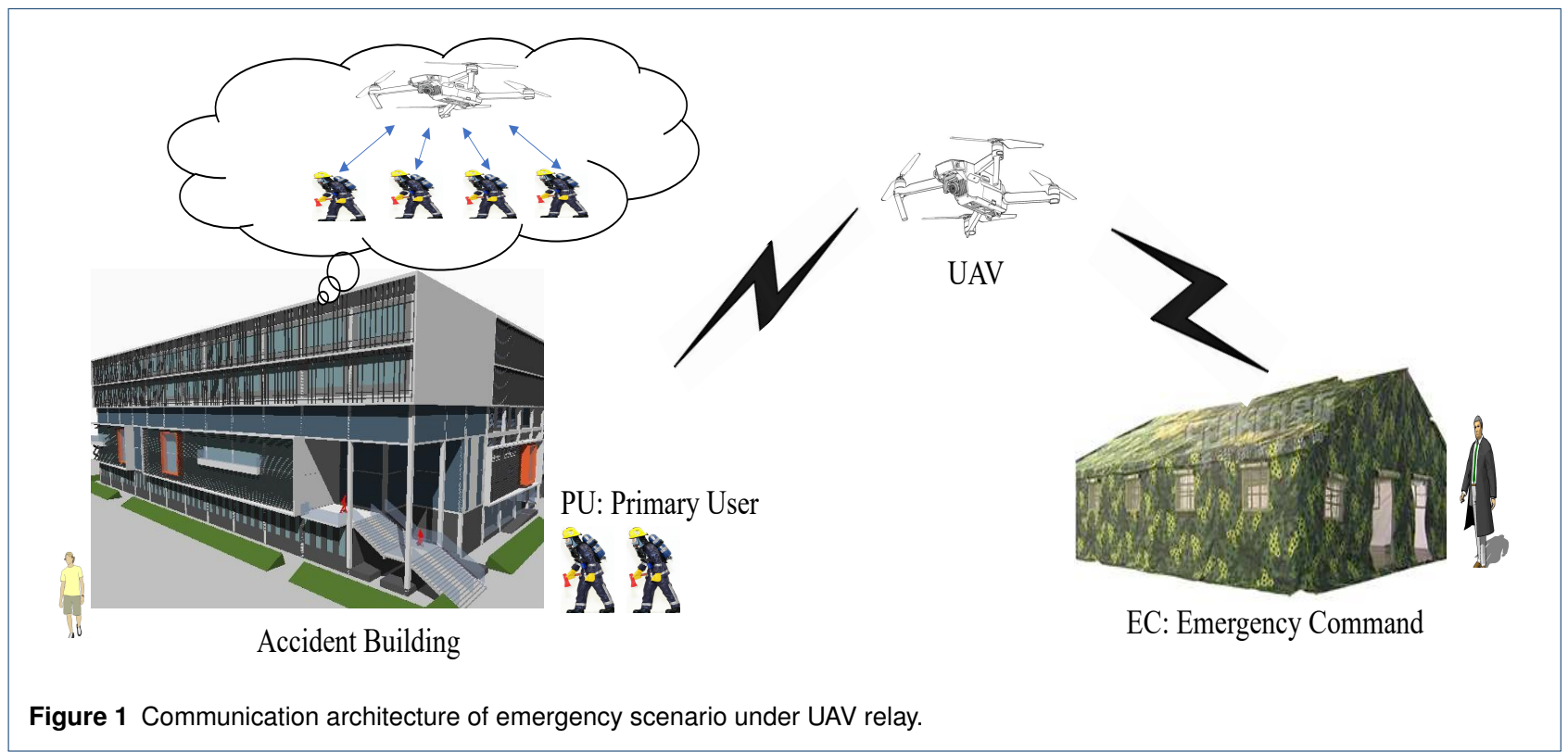

setting to ensure the detection success probability is the method to reduce the energy consumption of UAV.

\subsection{Spectrum sensing based on UAV}

In actual communication, communication signals generally go through signal processing processes such as sampling, quantization, coding, and modulation. After these processes, some digital characteristics of the signal have inherent periodicity, which is called cyclostationarity feature [?]. The noise has the characteristics of random stationarity but not cyclostationarity. It is an effective method to judge the existence of PU by analyzing the cyclostationarity of the received signal. Cyclostationarity feature detection is a method to detect primary user transmission by using cyclostationarity feature of the received signal. Cyclostationarity is caused by the periodicity of signal or its statistics such as mean and autocorrelation, which can be used to assist spectrum sensing.

\subsubsection{Cyclostationary feature detection}

In the disaster emergency communication environment, the digital characteristics of cyclostationary signals change with time cycle. This kind of signal is defined as:

$$
\left\{\begin{array}{l}
a_{x}(t)=a_{x}(t+n T)=E[x(t)] \\
R_{x}(t, \tau)=R_{x}(t+n T, \tau)=E\left[x(t) x^{*}(t+\tau)\right]
\end{array}\right.
$$

where $x(t)$ is continuous time signal, $a_{x}(t)$ is mean value, the autocorrelation function of $x(t)$ is $R_{x}(t, \tau), T$ is cycle period, $\tau$ is time latency.

It can be seen from equation (1) that $R_{x}(t, \tau)$ changes with time period. Assuming that the period is $T^{\prime}, R_{x}(t, \tau)$ can be expanded in the form of Fourier series:

$$
\begin{aligned}
R_{x}(t, \tau) & =\sum_{q}^{\infty} R_{x}^{a}(\tau) e^{j 2 \pi q t / T^{\prime}} \\
& =\sum_{a}^{\infty} R_{x}^{a}(\tau) e^{j 2 \pi a t}
\end{aligned}
$$

where $a$ is the cyclic frequency, $a=q / T^{\prime}, q$ is the integer, and $R_{x}^{a}(\tau)$ is the Fourier series coefficient, which can be expressed as:

$$
R_{x}^{a}(t, \tau)=\frac{1}{T^{\prime}} \int_{T^{\prime}}^{\infty} R_{x}(t, \tau) e^{-j 2 \pi a t} d t
$$

Cyclostationary signal satisfies ergodicity, and autocorrelation function can be obtained by sampling sample estimation [?]. The autocorrelation function can be expressed as:

$$
R_{x}(t, \tau)=\lim _{z \rightarrow \infty} \frac{1}{2 z+1} \sum_{n=-z}^{n=z} x\left(t+n T^{\prime}\right) x^{*}(t+n T+\tau)
$$

substituting equation (4) into equation (3), we can get the following results:

$$
R_{x}^{a}(\tau)=\lim _{T \rightarrow \infty} \frac{1}{T} \int_{T}^{\infty} x(t) x^{*}(t+\tau) e^{-j 2 \pi a t} d t
$$

where $R_{x}^{a}(\tau)$ is the cyclic autocorrelation function (CAF), and $R_{x}^{a}(\tau)$ has one more parameter $a$ than $R_{x}(t, \tau)$, which is called the cyclic frequency. The power spectral density of the signal is obtained by Fourier transform of $R_{x}(t, \tau)$. 
The same operation is applied to $R_{x}^{a}(\tau)$ to obtain the cyclic spectral density function as follows:

$$
S_{x}^{a}(f)=\int_{-\infty}^{+\infty} R_{x}^{a}(\tau) e^{-j 2 \pi f \tau} d \tau
$$

where $S_{x}^{a}(f)$ is the cyclic spectrum density (CSD) of the signal. When $a=0, R_{x}^{a}(\tau)$ and $S_{x}^{a}(f)$ show the stationary characteristic of the signal. When $a \neq 0, R_{x}^{a}(\tau)$ and $S_{x}^{a}(f)$ show the cyclostationary characteristic of the signal.

Based on $R_{x}^{a}(\tau)$ and $S_{x}^{a}(f)$, another judging condition of cyclostationary signal is given.

$$
\left\{\begin{array}{l}
\exists a \neq 0, R_{x}^{a}(\tau) \neq 0 \\
\exists a \neq 0, S_{x}^{a}(f) \neq 0
\end{array}\right.
$$

when equation (7) is satisfied, the signal is cyclostationary and its $S_{x}^{a}(f)$ is not zero at $a \neq 0$. For noise, it has no cyclostationary characteristic, that is to say, it satisfies the following requirements:

$$
\left\{\begin{array}{l}
\forall a \neq 0, R_{n}^{a}(\tau)=0 \\
\forall a \neq 0, S_{n}^{a}(f)=0
\end{array}\right.
$$

when $a=0, R_{n}^{a}(\tau)$ and $S_{n}^{a}(f)$ of noise are not 0 . Therefore, the existence of authorized user signal can be detected by the peak characteristic of cyclic spectral density function under $a \neq 0$.

The cyclic spectrum of signal can be analyzed by crosspower spectrum theory [?]. Let:

$$
\left\{\begin{array}{l}
l(t)=x(t) e^{-j 2 \pi \frac{a}{2} t} \\
m(t)=x(t) e^{j 2 \pi \frac{a}{2} t}
\end{array}\right.
$$

by Fourier transform of $l(t)$ and $m(t)$ in equation (9), the frequency domain expressions of $L(f)$ and $M(f)$ can be obtained according to the frequency shift characteristics

$$
\left\{\begin{array}{l}
L(t)=X(f+a / 2) \\
M(t)=X(f-a / 2)
\end{array}\right.
$$

According to equation (5) and equation (9), it can be concluded that:

$$
\begin{aligned}
R_{x}^{a}(\tau) & =\lim _{T \rightarrow \infty} \frac{1}{T} \int_{-T / 2}^{T / 2} l(t+\tau / 2) m^{*}(t-\tau / 2) d t \\
& =R_{l m}^{0}(\tau) .
\end{aligned}
$$

According to equation (11), $R_{x}^{a}(\tau)$ can be obtained by cross-correlation of signals $l(t)$ and $m(t)$. By substituting equation (11) into equation (6), we can get

$$
\begin{aligned}
S_{x}^{a}(f) & =\int_{-\infty}^{+\infty} R_{l m}^{0}(\tau) e^{-j 2 \pi f \tau} d \tau \\
& =S_{l m}^{0}(f)
\end{aligned}
$$

$S_{x}^{a}(f)$ can be obtained by calculating the cross-correlation density of $l(t)$ and $m(t)$. According to formula (9), it can be seen that $l(t)$ and $m(t)$ are obtained by $x(t)$ frequency shift $\pm a / 2$, that is, $S_{x}^{a}(f)$ is the correlation degree of signal at frequency component $f \pm a / 2$, so $S_{x}^{a}(f)$ is also called spectral correlation function.

\subsubsection{Cyclic spectral density analysis}

In this section, we will consider the method of realizing spectrum sensing in the actual environment. In the emergency communication scenario of this paper, for a continuous time signal in a frequency band, it is not to process the signal in the whole time domain. Generally, it will analyze and calculate the signal in a limited time period. This will greatly reduce the complexity of the algorithm, but also reduce the energy consumption of UAV. In the calculation of cyclic spectral density, the Fourier transform of signal $x(t)$ in a finite time period $\left[t^{\prime}-T / 2, t^{\prime}+T / 2\right]$ is as follows:

$$
X_{T}\left(t^{\prime}, f\right)=\int_{t^{\prime}-T / 2}^{t^{\prime}+T / 2} x(u) e^{-j 2 \pi f u} d u
$$

the following equation can be obtained from equation (10)

$$
\left\{\begin{array}{l}
L_{T}\left(t^{\prime}, f\right)=X_{T}\left(t^{\prime}, f+a / 2\right) \\
M_{T}\left(t^{\prime}, f\right)=X_{T}\left(t^{\prime}, f-a / 2\right) .
\end{array}\right.
$$

Combined with equations (11), (12) and (14), the cross spectral correlation function of $l(t)$ and $m(t)$ can be obtained. If we average cross spectral correlation function in time $\Delta t$, we can get

$$
S_{x_{T}}^{a}\left(t^{\prime}, f\right)_{\Delta t}=\frac{1}{\Delta t} \int_{-\Delta t / 2}^{\Delta t / 2} \frac{1}{T} L_{T}\left(t^{\prime}+h, f\right) M_{T}^{*}\left(t^{\prime}+h, f\right) d h
$$

the integral transformation is as follows:

$$
S_{x_{T}}^{a}\left(t^{\prime}, f\right)=\frac{1}{T} X_{T}\left(t^{\prime}, f+a / 2\right) X_{T}^{*}\left(t^{\prime}, f-a / 2\right) .
$$

Equation (16) is usually called cyclic periodogram. Although cyclic periodogram can also be used to estimate spectral correlation function directly, the error is large.

When $\Delta t \rightarrow \infty$ in equation (15), the expression of cyclic spectral correlation function in ideal state can be obtained as follows:

$$
S_{x}^{a}(f)=\lim _{T \rightarrow \infty} \lim _{\Delta t \rightarrow \infty} S_{x_{T}}^{a}\left(t^{\prime}, f\right)_{\Delta t}
$$

In practice, the cyclic periodogram is smoothed in order to get a better predicted value. Frequency smooth method (FSM), which is often used in the estimation of cyclic spectrum. In the frequency domain smoothing method, let 
$T=\Delta t$, and smooth equation (16) in frequency domain to obtain the estimation formula of cyclic spectral density function as follows:

$$
\begin{array}{r}
S_{x}^{a}\left(t^{\prime}, f\right)_{\Delta f}=\frac{1}{\Delta f} \int_{-\Delta f / 2}^{\Delta f / 2} \frac{1}{\Delta t} X_{\Delta t}\left(t^{\prime}, f+s+a / 2\right) \\
X_{\Delta t}^{*}\left(t^{\prime}, f+s-a / 2\right) d s
\end{array}
$$

where $\Delta t$ is the value of the limited time period of signal processing, the signal in the limited time period is uniformly sampled to obtain $N$ sampling points, and the sampling frequency is $F_{S}$, then the sampling interval is $f_{s}=F s / N$. For the discrete value $x\left(t^{\prime}, n\right)$ of the sampled signal, the discrete Fourier transform is obtained as follows

$$
X_{\Delta t}\left(t^{\prime}, k\right)=\sum_{n=0}^{N-1} x\left(t^{\prime}, n\right) e^{-j(2 \pi / N) n k} .
$$

Since the frequency range of signal is from negative frequency to positive frequency, it is necessary to approximate the discrete Fourier transform to the Continuous Fourier transform

$$
\left.X_{\Delta t}\left(t^{\prime}, k\right)\right|_{f=k f_{s}}=\left(1 / F_{s}\right) X_{\Delta t}\left(t^{\prime}, k\right)
$$

where:

$$
X_{\Delta t}\left(t^{\prime}, k\right)=\left\{\begin{array}{rr}
X_{\Delta t}\left(t^{\prime}, k\right), & k \geq 0 \\
X_{\Delta t}\left(t^{\prime}, k+N\right), & k<0 .
\end{array}\right.
$$

Let $M$ be the number of samples smoothed in the frequency domain and the frequency smoothing interval $\Delta f=$ $M f_{s}$. discrete the continuous frequencies in equation (18) to obtain the following equation:

$$
\begin{array}{r}
S_{x_{\Delta t}}^{a}\left(t^{\prime}, f\right)_{\Delta f}=\frac{1}{M N} \sum_{q=-\frac{M}{2}}^{\frac{M}{2}-1} X_{\Delta t}\left(t^{\prime}, f+a / 2+a f_{s}\right) \\
X_{\Delta t}^{*}\left(t^{\prime}, f-a / 2+q f_{s}\right) .
\end{array}
$$

Suppose $f=k f_{s}, a=2 h f_{s}$, then $k, h$ is the digital frequency of frequency $f$ and cycle frequency $a$ based on $f_{s}$, respectively. In practice, $t^{\prime}$ is fixed, so the final discrete form of formula (22) is as follows:

$$
S_{x_{\Delta t}}^{h}(f)_{\Delta t}=\frac{1}{M N} \sum_{q=-\frac{M}{2}}^{\frac{M}{2}-1} X_{\Delta t}(k+h+q) X_{\Delta t}^{*}(k-h+q) .
$$

After the above theoretical analysis and formula derivation of the frequency domain smoothing method for solving the cyclic spectral density function in the actual situation, the estimation algorithm of the cyclic spectral density function at the final discrete frequency can be obtained. The flow chart is shown in Figure 2.

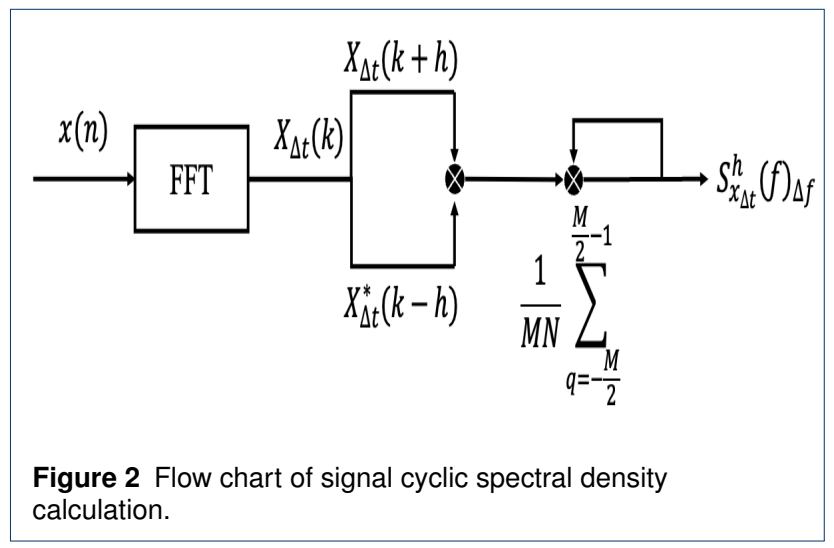

According to the fact that the modulated signal has cyclostationarity but the noise does not, the spectrum sensing model under cyclostationarity feature detection can be established according to equations (7) and (8). The equation is as follows: when $a=0$ :

$$
S_{R}^{a}(f)=\left\{\begin{array}{rr}
S_{n}^{a}(f), \quad H_{0} \\
S_{x}^{a}(f)+S_{n}^{a}(f), & H_{1} .
\end{array}\right.
$$

when $a \neq 0$ :

$$
S_{R}^{a}(f)=\left\{\begin{array}{rr}
0, & H_{0} \\
S_{x}^{a}(f), & H_{1}
\end{array}\right.
$$

From equation (24) and equation (25), when $a \neq 0$, $S_{n}^{a}(f)=0$, there is only $S_{x}^{a}(f)$ with value in the received signal. Therefore, when detecting whether there is an authorized user signal, we only need to observe the cyclic spectral density amplitude of the received signal at $a \neq 0$. If obvious peak characteristics are observed, it indicates that there is a primary user signal in the detected frequency band. Because there is $S_{n}^{a}(f)=0$ at $a \neq 0$, the cyclostationary feature detection algorithm can better suppress the impact of noise on the signal detection process.

\subsection{Relay based on UAV}

When rescuers are in a complex electromagnetic environment, the key of UAV relay communication is to relay the signals received by relay nodes. Different processing schemes lead to different cooperative communication protocols. The way of relay processing is different according to the relay forwarding protocol. Common relay and forward protocols include amplify and forward protocol, decode and forward protocol, and coding cooperation protocol. At the scene of rescue, no matter which protocol is 
used, communication may be outage. For emergency communication or temporary communication, rescue workers will prepare standby communication frequency before entering the disaster area. In the process of rescue, when the signal is outage suddenly due to interference, the rescuers switch the standby frequency. This switching process is considered to be random. In order to establish the communication between the rescue personnel and the headquarters, the UAV needs to carry out spectrum sense, find the standby frequency for the rescue personnel to switch, and then use the UAV as the relay to establish the communication between the rescue personnel and the headquarters. After the communication is established, in order to ensure that the communication is not outage, it is necessary to establish the relay probability model. For this process, the following communication relay probability model and communication relay sensing decision algorithm are established.

The communication channel between UAV and rescue personnel and emergency headquarters is A2G(Air to Ground) channel. There is a certain probability that the A2G channel is the line of sight channel or a non-line of sight channel, which depends on the environmental characteristics of the UAV communication scene and the elevation angle with ground terminal equipment. The communication channel between UAV and rescue personnel is a non-line of sight channel, and the communication channel between UAV and emergency command is a line of sight channel.

Let $i$ and $j$ denote the two nodes of each link. According to reference [?], the probability expression of A2G channel between $i$ and $j$ as line of sight (NoS) channel is

$$
P_{i j}^{L o S}=\frac{1}{1+\omega_{1} e^{-\omega_{2}\left(\theta_{i j}-\omega_{1}\right)}}
$$

the probability of non line of sight (NLoS) channel is

$$
P_{i j}^{N L o S}=1-P_{i j}^{L o S}
$$

where $\theta_{i j}$ is the elevation angle between the two points, $\omega_{1}$ and $\omega_{2}$ are the environmental characteristic parameters.

According to reference [?], the NLoS transmission component in the relay process between $i$ and $j$ is expressed as

$$
F_{i j}^{N L o S}=\frac{c^{2} d_{i j}^{-n}}{\left(4 \pi f_{c}\right)^{2}} P L_{i j}^{N L o S}
$$

where $c$ is the speed of light, $f_{c}$ is carrier frequency, $d_{i j}$ is the distance from the rescuer to the drone, $n$ is the path loss factor, $P L_{i j}^{N L O S}$ is the NLoS transmission component in the communication process from PU to UAV, $P L_{i j}^{N L o S}=$ $20 \lg \left(\frac{4 \pi f_{c} d_{i j}}{c}\right)+L_{N L o S} . L_{N L o S}$ is the average additional loss of the link.
The transmission component between $i$ and $j$ NoS can also be obtained

$$
F_{i j}^{N o S}=\frac{c^{2} d_{i j}^{-n}}{\left(4 \pi f_{c}\right)^{2}} P L_{i j}^{N o S}
$$

where $P L_{i j}^{N o S}=20 \lg \left(\frac{4 \pi f_{c} d_{i j}}{c}\right)+L_{N o S}, L_{N o S}$ is the average additional loss of the link.

The unless outage probability of a communication link is defined as the probability that the received SNR falls above a predetermined threshold $\lambda$. Therefore, the reliability probability $P$ of the communication from PU to the $\mathrm{EC}$ is shown as

$$
P=P\left(\frac{P_{i j}^{N L o S} F_{i j}^{N L o S}}{N_{0}}>\lambda\right) P\left(\frac{\left(1-P_{i j}^{N L o S}\right) F_{i j}^{L o S}}{N_{0}}>\lambda\right)
$$

where $N_{0}$ is the power of noise. According to the communication scenario in this paper and the scenario settings in the front of this section, the following equation is obtained by converting equation (30)

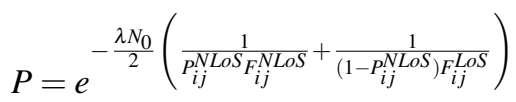

When doing spectrum sensing of UAV, the limit of interruption probability is added, and the power of UAV is adjusted at any time to meet the stability of the communication line. The following UAV Communication Relay sensing decision algorithm is established.

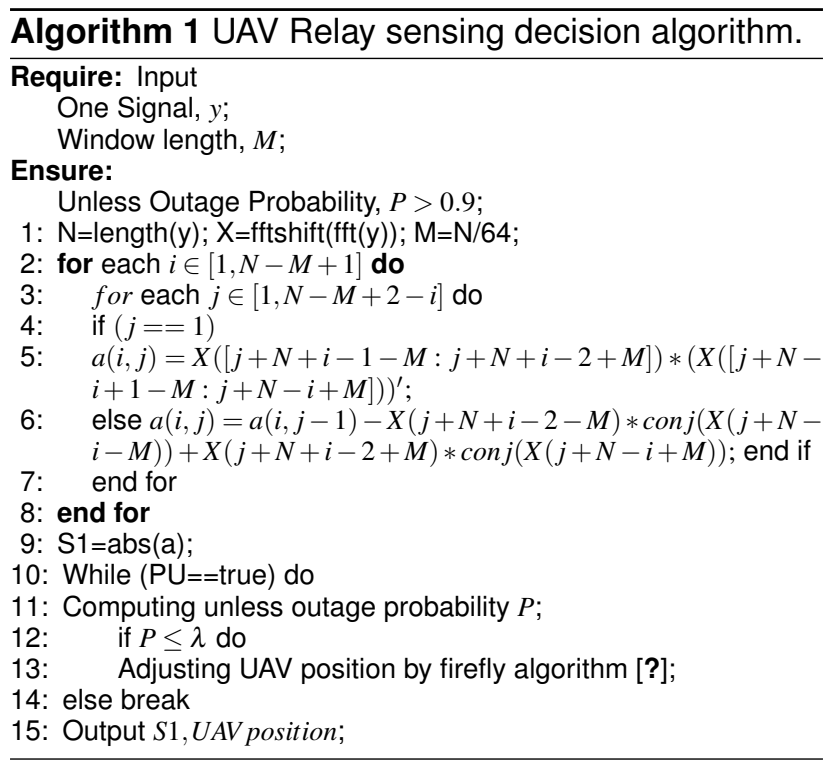

The algorithm includes the frequency domain smoothing algorithm in spectrum sensing, which is used to highlight 
the primary user signal in the signal, so as to prove whether a certain frequency is occupied by PU [?] [?]. It also adds the classic firefly algorithm to adjust the position of the UAV to ensure that the communication is not outage [?] [?]. Before using the firefly algorithm, spectrum sensing is carried out first, and the link will be evaluated only after sensing the existence of users, otherwise, the next signal will be detected.

When the UAV flies towards the PU, the sensing performance and throughput of the UAV system will be better. However, the energy consumed by its movement will be increased. Hence, a tradeoff exists between the flying distance and the energy optimization in CR-based UAV relay communication. In this section, the firefly algorithm is used to search the minimum value of the objective function to get the optimal location of the UAV. The objective function is as follows:

$$
\begin{array}{r}
\operatorname{Min} E_{p}=E_{1}+E_{2}+E 3 \\
\text { s.t. }: C_{1}, C_{2}, C_{3}, C_{4}
\end{array}
$$

where $E_{p}$ is the flight energy consumption in the process of UAV moving, $E_{1}$ is the spectrum sensing energy consumption of UAV, $E_{2}$ is the energy consumption of UAV communication. $C_{1}$ is the distance constraint on UAV's movement $d_{1}, C_{2}$ is the power constraint $G, C_{3}$ is the step size $S_{g}$ constraint on UAV's movement, and $C_{4}$ is spectrum sensing range constraint $d_{2}$.

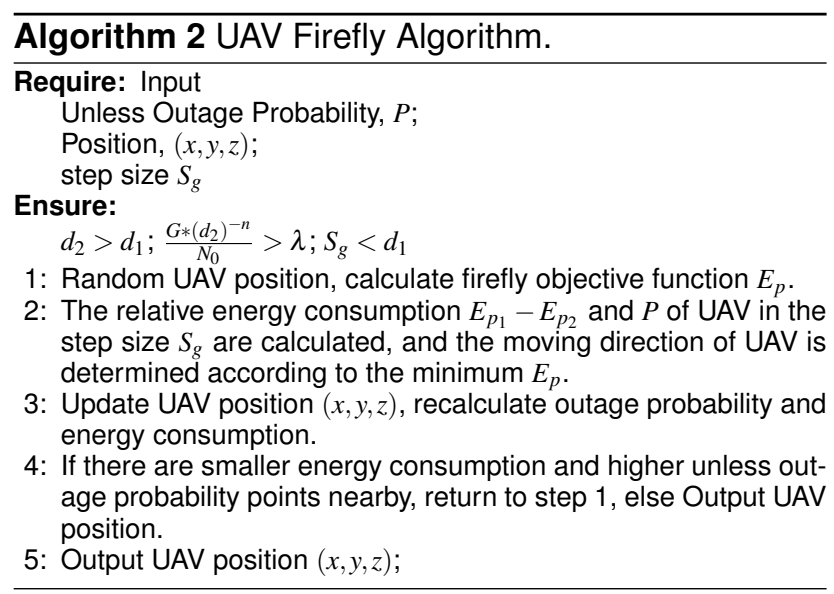

\section{RESULTS AND DISCUSSION}

In this paper, the double side band (DSB) signal under noise background is simulated, and the existence of detection signal is observed from its cyclic spectral density diagram. The Fig. 3 below is an analog DSB signal.

Set the carrier frequency $f_{c}=200 \mathrm{~Hz}, N=512$, sampling frequency $f_{S}=5120 \mathrm{~Hz}$ and signal-to-noise ratio $S N R=$ $2 d B$. The three-dimensional simulation diagram of cyclic spectral density of signal and its contour map are shown in Fig. 4 and 5

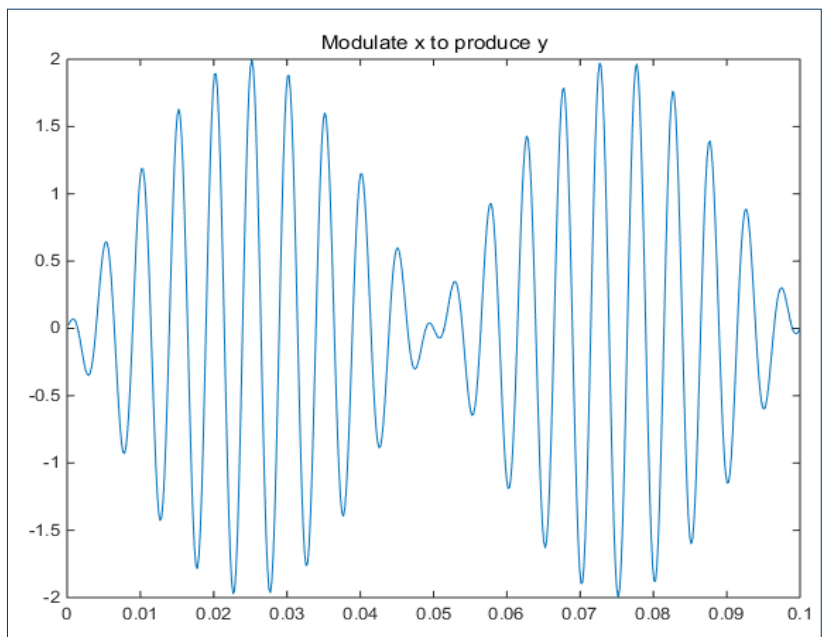

Figure 3 DSB signal.
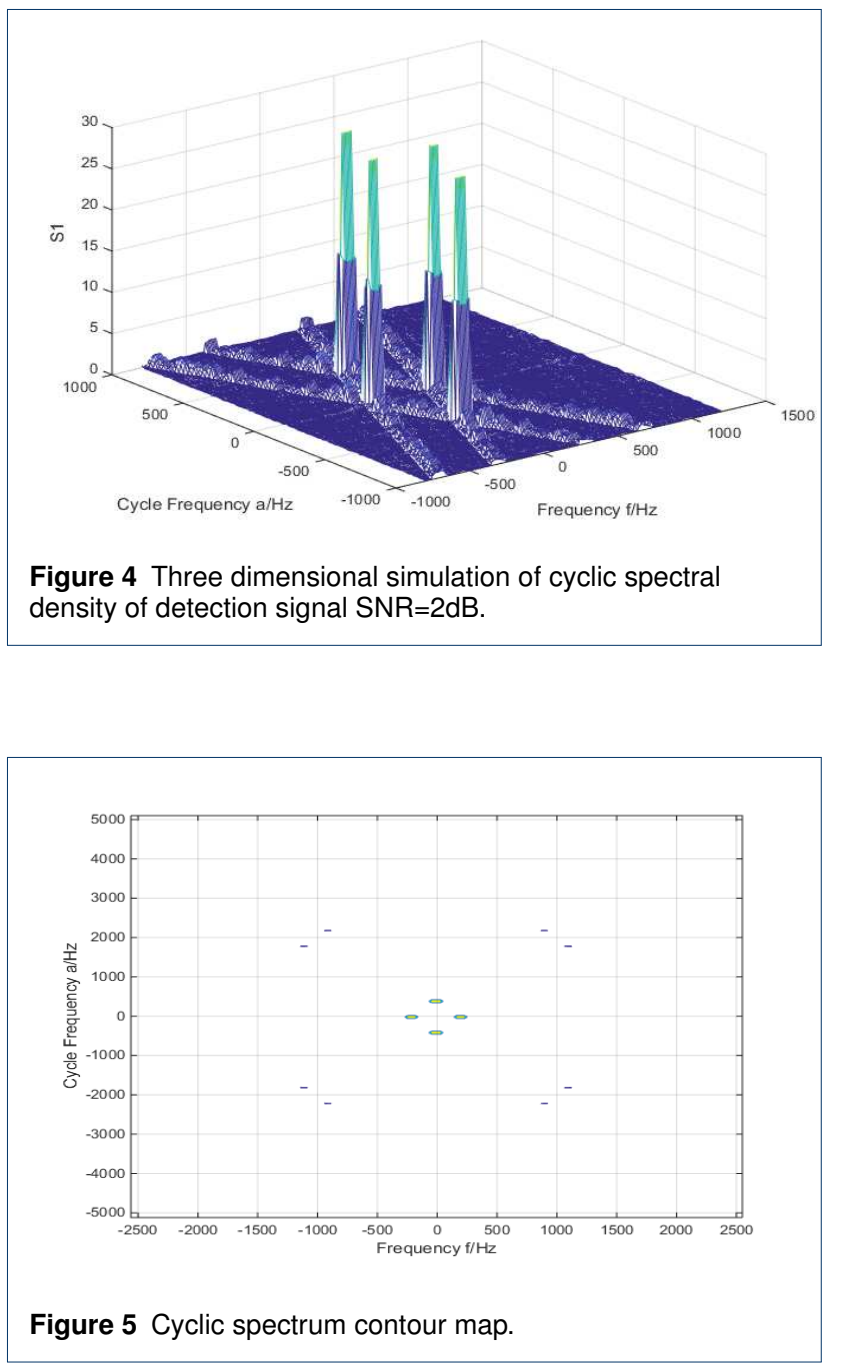
The cyclic spectral density of DSB signal has spectral component at $a=0$ and $\pm 2 f_{c}$. The existence of PU signal can be detected only by looking at whether the cyclic spectrum has a significant peak characteristic in $a= \pm 2 f_{c}$. According to Fig. 4 and Fig. 5, there is a spectrum component at $a= \pm 400 \mathrm{HZ}$, and there is a significant peak, that is, the PU signal exists.

In the estimation of cyclic spectral density, the calculation of signal and noise is a processing operation in finite time, so as to obtain the cyclic spectral density of received signal. At this time, when $a \neq 0$, the cyclic spectral density $S_{n}^{a}(f)$ of noise is no longer constant zero, but there is a certain amplitude value, and the amplitude value will increase the interference to the signal to a certain extent with the decrease of communication channel quality. Change the signal-to-noise ratio and observe the influence of signal-to-noise ratio. Set $S N R=-10 \mathrm{~dB}$, carrier frequency $f_{c}=200 \mathrm{~Hz}$, sampling points $N=512$ and sampling frequency $f_{s}=5120 \mathrm{~Hz}$ to get Fig. 6 .

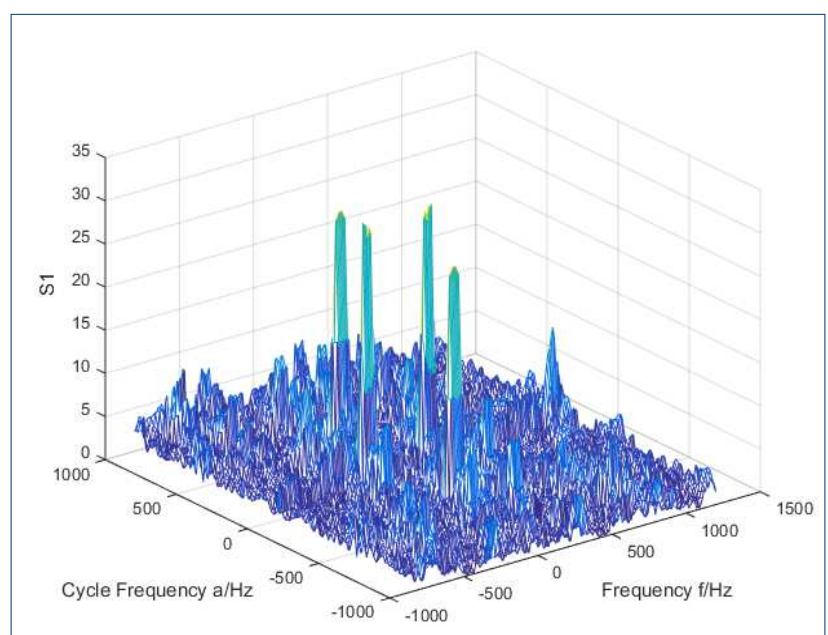

Figure 6 Three dimensional simulation of cyclic spectral density of detection signal SNR=-10dB.

According to Fig. 6, when the signal-to-noise ratio drops to $-10 d B$, the interference of noise to the signal is obviously enhanced, but the existence of the authorized user signal can be detected according to the graph outline. This also shows that the cyclostationary feature detection algorithm can reflect a certain anti-noise performance when the signal-to-noise ratio is small, but it will lose the ability to detect the signal when the signal-to-noise ratio is too small.

This shows that when the environmental noise is too large, the detection performance of UAV will decline. In order to detect the existence of users in the signal, there are two schemes. One way is increasing the detection range of the spectrum will increase the complexity of cycle detection, which will undoubtedly increase the energy consumption of UAV. The other way is moving the UAV's position makes the outage probability of signal access greater than the threshold $\lambda$.

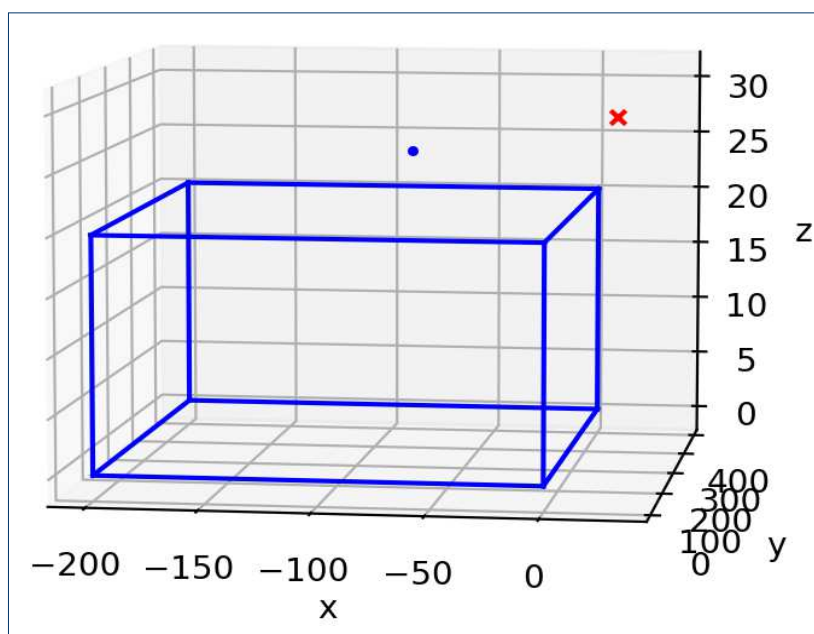

Figure 7 UAV position after doing firefly algorithm.

The simulation is aimed at a single UAV under the restriction of outage probability, using a firefly algorithm to adjust the position of the UAV until the end of the UAV Communication Relay sensing decision algorithm. Fig. 7 shows the location of the UAV after Algorithm 2. The red cross in Figure 7 is the best location point after the firefly algorithm search, and the blue point is the point where the UAV only considers the optimization of spectrum sensing.

\section{CONCLUSION}

Firstly, the spectrum sensing model of UAV is established, and the existence of PU is detected by a frequency-domain smoothing algorithm. Then, a UAV relay sensing decision algorithm is proposed based on the spectrum sensing algorithm. The goal of the UAV relay sensing decision algorithm is to adjust the position and ensure that the UAV relay link is not outage. Finally, the feasibility of the UAV relay sensing decision algorithm is verified by simulation, which integrates UAV spectrum sensing and position optimization. In the future, we will focus on the relationship among UAV energy, position, and perception, establish a system model and optimize UAV energy.

\section{Abbreviations}

UAV:Unmanned Aerial Vehicle; CR:cognitive radio; CNR:cognitive radio network; PER: primary exclusive region; 3D: three dimensional; RF:radio frequency;

\section{Acknowledgements}

This research is the result of the integration project of air, space and earth between Xi'an University and Beijing University of Posts and telecommunications

Funding

This work was supported in part by China Scholarship Council. 


\section{Availability of data and materials}

All data of simulation and experiment can be obtained by contacting the authors of the paper

\section{Competing interests}

The authors declare that they have no competing interests.

\section{Authors' contributions}

Both authors in the paper have equal contributions from the idea to the completion of the paper.

\section{Authors' information}

Zinan Guo is currently pursuing the Ph.D. degree with the State Key Laboratory of Networking and Switching Technology, Beijing University of Posts and Telecommunications, Beijing, China. His research interests are in the areas of UAV, and vehicle communication.

Liuying Wang ( lywang_3@stu.xidian.edu.cn) is currently pursuing the master degree with the State Key Laboratory of ISN, Xidian University, 710071, Xi'an, Shaanxi, China. Her research interests are in the areas of UAV, and Integration of sky and earth.

\section{Author details}

${ }^{1}$ Beijing University of Posts and Telecommunications Beijing, the State Key Laboratory of Networking and Switching Technology, Beijing, China. ${ }^{2}$ State Key Laboratory of ISN, Xidian University, 710071, Xi'an, Shaanxi, China.

\section{References}

1. Li, S., Duo, B., Yuan, X., Liang, Y., Di Renzo, M.: Reconfigurable intelligent surface assisted uav communication: Joint trajectory design and passive beamforming. IEEE Wireless Communications Letters 9(5), 716-720 (2020). doi:10.1109/LWC.2020.2966705

2. Chen, X., Hu, X., Zhu, Q., Zhong, W., Chen, B.: Channel modeling and performance analysis for uav relay systems. China Communications 15(12), 89-97 (2018). doi:10.12676/j.cc.2018.12.007

3. Deng, L., He, Y., Liu, Q.: Research on application of fire uumanned aerial vehicles in emergency rescue. In: 2019 9th International Conference on Fire Science and Fire Protection Engineering (ICFSFPE), pp. 1-5 (2019). doi:10.1109/ICFSFPE48751.2019.9055875

4. leee standard - information technology-telecommunications and information exchange between systems-wireless regional area networks-specific requirements-part 22: Cognitive wireless ran mac and phy specifications: Policies and procedures for operation in the bands that allow spectrum sharing where the communications devices may opportunistically operate in the spectrum of primary service. IEEE Std 802.22-2019 (Revision of IEEE Std 802.22-2011), 1-1465 (2020). doi:10.1109/IEEESTD.2020.9086951

5. Chen, K.-C., Prasad, R.: Spectrum Management of Cognitive Radio Networks, pp. 335-355 (2009). doi:10.1002/9780470742020.ch11

6. Sawant, R., Nema, S.: On performance of an outage probability of hybrid cooperative cognitive radio networks with rayleigh faded networks. In: 2020 International Conference on Industry 4.0 Technology (I4Tech), pp. 18-22 (2020). doi:10.1109/14Tech48345.2020.9102702

7. Mei, W., Zhang, R.: Uav-sensing-assisted cellular interference coordination: A cognitive radio approach. IEEE Wireless Communications Letters 9(6), 799-803 (2020). doi:10.1109/LWC.2020.2970416

8. Yoshikawa, K., Yamamoto, K., Nishio, T., Morikura, M.: Grid-based exclusive region design for $3 d$ uav networks: A stochastic geometry approach. IEEE Access 7, 103806-103814 (2019). doi:10.1109/ACCESS.2019.2931344

9. Ji, B., Li, Y., Cao, D., Li, C., Mumtaz, S., Wang, D.: Secrecy performance analysis of uav assisted relay transmission for cognitive network with energy harvesting. IEEE Transactions on Vehicular Technology 69(7), 7404-7415 (2020). doi:10.1109/TVT.2020.2989297

10. Ji, B., Li, Y., Chen, S., Han, C., Li, C., Wen, H.: Secrecy outage analysis of uav assisted relay and antenna selection for cognitive network under nakagami- $m$ channel. IEEE Transactions on Cognitive Communications and Networking 6(3), 904-914 (2020). doi:10.1109/TCCN.2020.2965945

11. Tığlı, B., Erdem, E., Erdoğan, E.: Outage probability performance of cognitive radio enabled uav relaying. In: 2020 28th Signal Processing and Communications Applications Conference (SIU), pp. 1-4 (2020). doi:10.1109/SIU49456.2020.9302131

12. Khalifa, A., Kermorgant, O., Dominguez, S., Martinet, P.: Platooning of car-like vehicles in urban environments: An observer-based approach considering actuator dynamics and time delays. IEEE Transactions on Intelligent Transportation Systems, 1-13 (2020)

13. Godbole, D.N., Lygeros, J.: Longitudinal control of the lead car of a platoon. IEEE Transactions on Vehicular Technology 43(4), 1125-1135 (1994)

14. Wei, S., Zou, Y., Zhang, X., Zhang, T., Li, X.: An integrated longitudinal and lateral vehicle following control system with radar and vehicle-to-vehicle communication. IEEE Transactions on Vehicular Technology 68(2), 1116-1127 (2019)

15. Bayuwindra, A., Ploeg, J., Lefeber, E., Nijmeijer, H.: Combined longitudinal and lateral control of car-like vehicle platooning with extended look-ahead. IEEE Transactions on Control Systems Technology 28(3), 790-803 (2020)

16. Chehardoli, H., Ghasemi, A.: Adaptive centralized/decentralized control and identification of 1-d heterogeneous vehicular platoons based on constant time headway policy. IEEE Transactions on Intelligent Transportation Systems 19(10), 3376-3386 (2018). doi:10.1109/TITS.2017.2781152

17. Li, S.E., Zheng, Y., Li, K., Wang, J.: An overview of vehicular platoon control under the four-component framework. In: 2015 IEEE Intelligent Vehicles Symposium (IV), pp. 286-291 (2015). doi:10.1109/IVS.2015.7225700

18. Hao, H., Barooah, P., Mehta, P.G.: Stability margin scaling laws for distributed formation control as a function of network structure. IEEE Transactions on Automatic Control 56(4), 923-929 (2011). doi:10.1109/TAC.2010.2103416

19. Peng, J.Z.: Range policy of adaptive cruise control vehicles for improved flow stability and string stability. IEEE Transactions on Intelligent Transportation Systems 6(2), 229-237 (2005). doi:10.1109/TITS.2005.848359

20. Liang, C., Peng, H.: Optimal adaptive cruise control with guaranteed string stability. VEHICLE SYSTEM DYNAMICS 32(4-5), 313-330 (1999). doi:10.1076/vesd.32.4.313.208

21. Ghasemi, A., Kazemi, R., Azadi, S.: Stable decentralized control of a platoon of vehicles with heterogeneous information feedback. IEEE Transactions on Vehicular Technology 62(9), 4299-4308 (2013)

22. Yadlapalli, S.K., Darbha, S., Rajagopal, K.R.: Information flow and its relation to stability of the motion of vehicles in a rigid formation. IEEE Transactions on Automatic Control 51(8), 1315-1319 (2006)

23. Sadraddini, S., Sivaranjani, S., Gupta, V., Belta, C.: Provably safe cruise control of vehicular platoons. IEEE Control Systems Letters 1(2), 262-267 (2017)

24. Zheng, Y., Eben Li, S., Wang, J., Cao, D., Li, K.: Stability and scalability of homogeneous vehicular platoon: Study on the influence of information flow topologies. IEEE Transactions on Intelligent Transportation Systems 17(1), 14-26 (2016). doi:10.1109/TITS.2015.2402153

25. Li, S.E., Zheng, Y., Li, K., Wu, Y., Hedrick, J.K., Gao, F., Zhang, H.: Dynamical modeling and distributed control of connected and automated vehicles: Challenges and opportunities. IEEE Intelligent Transportation Systems Magazine 9(3), 46-58 (2017). doi:10.1109/MITS.2017.2709781

26. Rajamani, R., Han-Shue Tan, Boon Kait Law, Wei-Bin Zhang: Demonstration of integrated longitudinal and lateral control for the operation of automated vehicles in platoons. IEEE Transactions on Control Systems Technology 8(4), 695-708 (2000). doi:10.1109/87.852914

27. Ali, A.: Modeling and control of a platoon of urban autonomous vehicles. PhD thesis, :Research Institute in Communications and Cybernetics of Nantes (IRCCyN ) (2015)

28. M. Segata, H.M.C. T. R. Li: Safe and efficient communication protocols for platoon control. PhD Thesis (Dissertation) University of Innsbruck (2016)

29. Segata, M., Joerer, S., Bloessl, B., Sommer, C., Dressler, F., Cigno, R.L.: Plexe: A platooning extension for veins. In: 2014 IEEE Vehicular Networking Conference (VNC), pp. 53-60 (2014). doi:10.1109/VNC.2014.7013309 
30. Wang, S..D.W..v.A.B..S.B. M ; Hoogendoorn: Delay-compensating strategy to enhance string stability of adaptive cruise controlled vehicles. TRANSPORTMETRICA B-TRANSPORT DYNAMICS 6(3), 211-229 (2018). doi:10.1080/21680566.2016.1266973

31. Wu, H.-x., Cheng, S.-k., Cui, S.-m.: Communication of vehicle management unit in the electric vehicle. IEEE Transactions on Magnetics 41(1), 514-517 (2005). doi:10.1109/TMAG.2004.839273

32. Zander, J.: Performance of optimum transmitter power control in cellular radio systems. IEEE Transactions on Vehicular Technology 41(1), 57-62 (1992). doi:10.1109/25.120145

33. Grandhi, S.A., Vijayan, R., Goodman, D.J., Zander, J.: Centralized power control in cellular radio systems. IEEE Transactions on Vehicular Technology 42(4), 466-468 (1993). doi:10.1109/25.260766

34. Rosberg, Z., Zander, J.: Toward a framework for power control in cellular systems. Wireless Networks 4(4), 215-222 (1998)

35. Wei, L., Hu, R.Q., Qian, Y., Wu, G.: Energy efficiency and spectrum efficiency of multihop device-to-device communications underlaying cellular networks. IEEE Transactions on Vehicular Technology 65(1), 367-380 (2016). doi:10.1109/TVT.2015.2389823

36. 3GPP: 3rd generation partnership project 38.901 ; technical specification group radio access network; study on channel model for frequencies from 0.5 to $100 \mathrm{ghz}$ (release 16). (2019)

37. Sommer, C.: Veins. The open source vehicular network simulation framework (2006-2020)

38. Segata, M.: Plexe. The Platooning Extension for Veins (2014-2018)

39. Swaroop, D., Hedrick, J.K.: String stability of interconnected systems In: Proceedings of 1995 American Control Conference - ACC'95, vol. 3, pp. 1806-18103 (1995). doi:10.1109/ACC.1995.531196

40. Santini, S., Salvi, A., Valente, A.S., PescapšŠ, A., Segata, M., Lo Cigno, R.: A consensus-based approach for platooning with intervehicular communications and its validation in realistic scenarios. IEEE Transactions on Vehicular Technology 66(3), 1985-1999 (2017) doi:10.1109/TVT.2016.2585018

41. Hammache, M., Michaelian, M., Browand, F.: Aerodynamic forces on truck models including two trucks in tandem. SAE Paper 1(0530) (2002) 